Case Report

\title{
Novel Therapies for Myocardial Irritability following Extreme Hydroxychloroquine Toxicity
}

\author{
Paul B. McBeth, Perseus I. Missirlis, Harry Brar, and Vinay Dhingra \\ Division of Critical Care Medicine, University of British Columbia, Vancouver General Hospital, Vancouver, BC, Canada V5Z 1 M9 \\ Correspondence should be addressed to Paul B. McBeth; pmcbeth@gmail.com
}

Received 2 June 2015; Accepted 3 August 2015

Academic Editor: Serdar Kula

Copyright (C) 2015 Paul B. McBeth et al. This is an open access article distributed under the Creative Commons Attribution License, which permits unrestricted use, distribution, and reproduction in any medium, provided the original work is properly cited.

\begin{abstract}
Introduction. Hydroxychloroquine (HCQ) overdose is rare and potentially deadly when consumed in large doses. Management of severe HCQ toxicity is limited and infrequently reported. This report presents the case of a massive ingestion of HCQ. Case Report. A 23-year-old female presents following an intentional ingestion of approximately $40 \mathrm{~g}$ of HCQ. Within six hours after ingestion, she developed severe hemodynamic instability resulting from myocardial irritability with frequent ventricular ectopic activity leading to runs of polymorphic ventricular tachycardia (PMVT) and ventricular fibrillation (VF) requiring multiple defibrillations. Additional treatments included intravenous diazepam, epinephrine, norepinephrine, sodium bicarbonate, and magnesium sulfate. Despite the ongoing hemodynamic instability, the patient was also treated with Intralipid (ILE) and received hemodialysis. Improvements in her hemodynamics were observed after 18 hours. She survived her massive overdose of HCQ. Conclusion. HCQ poisoning is rare but serious because of its rapid progression to life-threatening symptoms. Hemodynamic support, gastric decontamination, electrolyte monitoring and replacement, and management of arrhythmias are the mainstays of treatment. The combined role of dialysis and ILE in the setting of massive HCQ overdose may improve outcomes.
\end{abstract}

\section{Introduction}

Hydroxychloroquine (HCQ) overdose is rare and often lethal when ingested in large doses. There exists a paucity of data on management of HCQ toxicity as it is infrequently reported. The majority of treatment recommendations are extrapolated from chloroquine (CQ) poising $[1,2]$. Current management strategies are targeted at myocardial stabilization, hemodynamic support, electrolyte correction, and decontamination $[1,2]$. We herein report a unique case of a massive $(\sim 40 \mathrm{~g})$ ingestion of HCQ complicated by coma, hemodynamic instability, and respiratory failure treated with mechanical ventilation, vasopressor, and inotropic support, as well as hemodialysis and intravenous lipid emulsion (Intralipid) (ILE).

\section{Case Presentation}

2.1. Clinical Presentation. A 23-year-old female with a past medical history of depression, borderline personality disorder, obsessive compulsive disorder, psychosis NOS, and congenital hydrocephalus with VP shunt presents following ingestion of approximately $40 \mathrm{~g}$ of HCQ. She had been using HCQ for the treatment of pruritus. She was also being treated with risperidone, clonazepam, and zopiclone for her psychiatric disorder and fluconazole at the time of presentation for the treatment of a vaginal yeast infection. At presentation, she had a Glasgow Coma Score (GCS): 15/15, temperature: $37.1^{\circ} \mathrm{C}$, blood pressure (BP): $92 / 60 \mathrm{mmHg}$, pulse rate: $65 / \mathrm{min}$, respiratory rate: $16 / \mathrm{min}$, blood glucose: $3.9 \mathrm{mmol} / \mathrm{L}$, and serum potassium: $3.0 \mathrm{mmol} / \mathrm{L}$. The initial blood gas demonstrated a metabolic acidosis and respiratory alkalosis: $\mathrm{pH}: 7.33, \mathrm{pCO}_{2}: 16, \mathrm{pO}_{2}: 70$, and bicarbonate: 8. Electrocardiograph performed on arrival demonstrated sinus rhythm with a widened QRS $(140 \mathrm{~ms})$ and QT interval (QTc $576 \mathrm{~ms}$ ). See Figure 1. Her urine toxicology was negative for salicylates, ethanol, acetaminophen, benzodiazepines, cannabinoid, cocaine, opiates, and amphetamines. The presenting renal and hepatic laboratory values are as follows: creatinine $(\mathrm{Cr}): 133 \mu \mathrm{mol} / \mathrm{L}$; albumin: $39 \mathrm{~g} / \mathrm{L}$; lipase: 113 units/L; gamma-glutamyl transpeptidase (GGT): 30 units/L; alkaline phosphatase: 55 units/L; total bilirubin: 


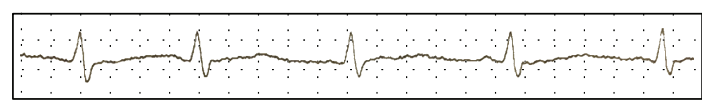

FIGURE 1: Presenting ECG tracing.

$3 \mu \mathrm{mol} / \mathrm{L}$; direct bilirubin: $1 \mu \mathrm{mol} / \mathrm{L}$; alanine aminotransferase (ALT): 23 units/L; aspartate aminotransferase (AST): 14 units/L.

2.2. Resuscitation and Management. Within three hours of her overdose, the patient became obtunded requiring intubation and mechanical ventilation. Hypotension was treated with crystalloid and vasopressor support. Gastric lavage and activated charcoal for decontamination were provided. Within six hours of her overdose, she demonstrated severe hemodynamic instability resulting from myocardial irritability with frequent ventricular ectopic activity leading to runs of polymorphic ventricular tachycardia (PMVT) and ventricular fibrillation (VF) requiring 18 defibrillations with 200J with biphasic defibrillator over the first 18 hours after ingestion. Given her protracted cardiac instability, additional treatments including intravenous diazepam $60 \mathrm{mg}$ followed by infusion of $6 \mathrm{mg} / \mathrm{hr}$ for calcium channel stabilization (total of $82 \mathrm{mg}$ given), epinephrine $20 \mathrm{mcg} / \mathrm{min}$, norepinephrine $30 \mathrm{mcg} / \mathrm{min}$, sodium bicarbonate $30 \mathrm{mmol} / \mathrm{hr}$, and magnesium sulfate $1 \mathrm{~g} / \mathrm{hr}$ infusions were also initiated. The serum potassium three hours after the overdose was $1.5 \mathrm{mmol} / \mathrm{L}$. In total, $380 \mathrm{mmol}$ of potassium chloride was given over the first 18 hours administered in $20 \mathrm{mmol}$ boluses. She also received intravenous sodium bicarbonate for alkalization. To treat refractory ventricular ectopy, a bolus dose of $1.5 \mathrm{~mL} / \mathrm{kg}$ of $20 \%$ Intralipid ILE was initially given followed by a high dose infusion over approximately 30 minutes. A total of $500 \mathrm{~mL}$ of ILE was given. No further infusion of ILE was given after this initial dose. Vascular access for dialysis was established immediately after the bolus dose and thus dialysis overlapped briefly with the infusion of ILE. The runs of PMVT and VF were felt to be secondary to a preexisting channelopathy likely potentiated by fluconazole and risperidone. Ventricular pacing was reviewed but not considered indicated. The patient was started on piperacillin/tazobactam for possible aspiration pneumonia. Echocardiographic imaging revealed normal global ventricular contractility with an ejection fraction of $60 \%$.

The patient was started on intermittent hemodialysis (IHD) in an attempt to reduce HCQ levels despite a paucity of literature regarding its use. The pharmacokinetics suggests that much of the drug is not accessible to the dialyzer, but given the severity of the overdose and the impact of hemodynamics even a small amount removed was considered favorable. Plasma HCQ level was 6425 mol/L 12 hours after ingestion. A five-hour run of hemodialysis was initiated with end points being either improved hemodynamics or of significant decrease in blood levels. A F1000 Dialyzer was used with a dialysate flow rate of $800 \mathrm{cc} / \mathrm{hr}$. In total, $135 \mathrm{~L}$ of blood was processed. As noted above, concurrent with the dialysis run, an ILE infusion was administered in an effort to create a "lipid sink" to sequester lipophilic HCQ. At 31 hours after ingestion, the plasma HCQ level was $4328 \mathrm{~mol} / \mathrm{L}$.

Improvements in her hemodynamics were observed after administration of dialysis and ILE. She was subsequently weaned from the ventilator and extubated on day 3 and discharged from ICU on postadmission day six.

\section{Discussion}

Hydroxychloroquine is sold under the trade name Plaquenil and is an aminoquinoline derivative used in the prophylaxis and treatment of malaria. It is also used as an antiinflammatory agent in rheumatoid arthritis and complications of lupus and connective tissue disorders with an off label use in the treatment of urticaria. Hydroxychloroquine is highly toxic in overdose resulting in rapid onset of hypotension, ventricular dysrhythmias, and cardiac arrest resulting in death [1-3]. Seizures, coma, and respiratory arrest can occur in patients with severe toxicity [3].

Descriptions of HCQ overdose are limited to case reports in the literature. Given this paucity of data, the current emergency treatment is modeled on the experience of CQ overdose. The mortality rate of a CQ overdose for adults is between 10 and $30 \%$ [1]. Unlike a CQ overdose, there is no established lethal or toxic dose of HCQ. Treatments are targeted at myocardial stabilization, hemodynamic support, electrolyte correction, decontamination, and prevention of seizures. At present, there is limited data regarding the use of dialysis and to our knowledge there is no description of the use of ILE in combination with dialysis.

Hydroxychloroquine has a dose-related cardiac sodium and potassium channel blocking effect resulting in delayed repolarization and slow intraventricular conduction. This results in bradycardia, hypotension, ventricular dysrhythmias, widened QRS, and prolonged QT interval [4]. Hypokalemia appears to be due to intracellular movement of potassium via a direct effect on cell membrane.

Hydroxychloroquine is rapidly absorbed following ingestion. Peak plasma levels of HCQ occurred 2-4.5 hours after ingestion. In overdose, onset of symptoms usually occurs within 30 minutes. It is highly tissue bound with a large volume of distribution. Distributional half-life is 15-30 hours [5]. Elimination half-life ranges from 4 to 40 days. Death from cardiorespiratory arrest or refractory shock often occurs within 3 hours after ingestion. ECG changes include QRS widening and QT prolongation $[3,6]$. Common dysrhythmias include ventricular ectopic beats, ventricular tachycardia, ventricular fibrillation, and torsades de pointes.

Patients presenting to health care facilities less than onehour after ingestion should be considered for gastric decontamination with gastric lavage and activated charcoal. The use of multiple-dose activated charcoal should be considered in severe HCQ poisoning [7, 8].

Profound hypokalemia is a known effect of HCQ poisoning and appears to correlate with toxicity. The mechanism is related to reduced potassium efflux from the blockade of membrane channels. It is unknown whether HCQ causes direct cardiotoxicity or if it is partly due to the hypokalemia. 
ECG findings include prolonged QT and QRS intervals leading to life-threatening ventricular arrhythmias and cardiac arrest [3, 6]. Aggressive potassium replacement is required; however, monitoring for rebound hyperkalemia [9-11] with resultant dysrhythmias is important. Diazepam infusion (1$2 \mathrm{mg} / \mathrm{kg}$ IV over 30 minutes) has been suggested to modify cardiac toxicity and improve survival based on animal experimental data $[4,12]$. This has also been demonstrated in several retrospective studies in patients with acute HCQ toxicity [1214]. The chronic use of HCQ may lead to QT prolongation [15]. Physicians prescribing HCQ to patients for extended use should consider monitoring patients for cardiac arrhythmias.

The clinical value of hemodialysis and peritoneal dialysis has not been established for the overdose of HCQ. The apparent volume of distribution for HCQ is approximately $600 \mathrm{~L} / \mathrm{kg}$. This implies that the drug rapidly becomes unavailable for removal after ingestion [1]. The extensive sequestration of HCQ by tissues limits effectiveness of hemodialysis. Despite the lack of guidelines in favor of hemodialysis in this case, it may have been helpful for the following reasons. CQ and by extension HCQ are known to interfere with potassium efflux and facilitate insulin release [16]. Hypokalemia and hypoglycemia are both known precipitants of cardiac arrest. Perhaps critical hypokalemia and hypoglycemia were averted by stabilizing plasma levels with hemodialysis. Secondly, in the face of the overwhelming overdose, the "lipid sink" provided by ILE may become saturated and thus abolish the gradient for lipophilic compounds to diffuse out from the intracellular compartment to the extracellular compartment. It is conceivable that the "lipid sink" diffusion gradient was preserved by dialysing Intralipid that had become saturated with HCQ. Extracorporeal membrane oxygenation (ECMO) has also been successfully demonstrated in an isolated case severe HCG toxicity [17]. The use of ECMO was not considered in this case.

Intravenous lipid emulsion is commercially available and termed Intralipid for treatment of local anesthetic systemic toxicity (LAST). Early basic science research by Weinberg et al. established that lipid emulsion successfully resuscitated rats and dogs from bupivacaine induced cardiac arrest [18, 19]. This work described the "lipid sink" theory of ILE, whereas ILE may act as an expanded lipid reservoir to sequester lipophilic bupivacaine away from cardiac myocytes [18]. Rosenblatt et al. described the first use of ILE for the treatment of cardiac arrest after bupivacaine and mepivacaine overdose [20]. Subsequently, Litz et al. reported the recovery of a perfusing cardiac rhythm with ILE after prolonged asystolic arrest following a ropivacaine overdose after axillary plexus block [21]. Given these promising developments, the role of ILE was expanded from the treatment of LAST to other toxic overdoses [17, 22]. Similar to bupivacaine, HCQ is lipophilic with a large volume of distribution [23] and blocks sodium channel function [24]. Experience with HCQ overdose is limited as there are few published case reports in the literature. Thus, resuscitation of HCQ overdose is managed similar to CQ overdose given their structural and toxidromal similarities [1]. A recent case report describes two cases of HCQ overdose in which ILE was utilized in the resuscitation [25]. Despite the standard resuscitation of these patients with sodium bicarbonate and diazepam, both died despite ILE infusion. The first case was a mixed overdose of HCQ and CQ in which the patient developed torsades des pointes that failed to respond to single bolus dose of $100 \mathrm{~mL}$ of $20 \%$ Intralipid. The second patient suffered from a cardiac arrest following a $20 \mathrm{mg}$ HCQ overdose with the return of spontaneous circulation (ROSC) after cardiopulmonary resuscitation (CPR) for 5 minutes. A bolus dose of $100 \mathrm{~mL}$ of 10 Intralipid was given followed by $400 \mathrm{~mL}$ over 30 minutes. The patient subsequently developed wide complex tachycardia and cardiac arrest with ROSC after 25 minutes of CPR. Despite these interventions, the patient suffered another arrest and died. In both cases, the authors did not institute hemodialysis.

\section{Conclusion}

In conclusion, HCQ poisoning is rare but serious because of its rapid progression to life-threatening symptoms. Hemodynamic support, gastric decontamination, electrolyte monitoring and replacement, and management of arrhythmias are the mainstays of treatment. The combined role of dialysis and ILE in the setting of massive HCQ overdose may improve outcomes by extending the "lipid sink" effect of ILE and normalizing electrolyte concentrations.

\section{Conflict of Interests}

The authors declare that there is no conflict of interests.

\section{Authors' Contribution}

Paul B. McBeth, Perseus I. Missirlis, and Harry Brar were major contributors in writing the paper, providing the revisions, and creating the figures. Paul B. McBeth, Harry Brar, Perseus I. Missirlis, and Vinay Dhingra provided revisions and contributed to the writing and completion of the paper. Vinay Dhingra provided critical revisions and gave final approval of the version for publication. All the contributing authors have read and approved the final paper.

\section{References}

[1] K. Marquardt and T. E. Albertson, "Treatment of hydroxychloroquine overdose," American Journal of Emergency Medicine, vol. 19, no. 5, pp. 420-424, 2001.

[2] P. Jordan, J. G. Brookes, G. Nikolic, and D. G. Le Counteur, "Hydroxycholoroquine overdose: toxicokinetics and management," Journal of Toxicology. Clinical toxicology, vol. 37, no. 7, pp. 861-864, 1997.

[3] G. K. Isbister, A. Dawson, and I. M. Whyte, "Hydroxychloroquine overdose: a prospective case series," American Journal of Emergency Medicine, vol. 20, no. 4, pp. 377-378, 2002.

[4] S. A. Pruchnicki, T. F. Good, and P. D. Walson, "Severe hydroxychloroquine poisoning reversed with diazepam," Journal of Toxicology. Clinical Toxicology, vol. 33, p. 582, 1996.

[5] D. N. Bateman, P. G. Blain, K. W. Woodhouse et al., "Pharmacokinetics and clinical toxicity of quinine overdosage: lack 
of efficacy of techniques intended to enhance elimination," Quarterly Journal of Medicine, vol. 54, no. 214, pp. 125-131, 1985.

[6] J.-L. Clemessy, C. Favier, S. W. Borron, P. E. Hantson, E. Vicaut, and F. J. Baud, "Hypokalaemia related to acute chloroquine ingestion," The Lancet, vol. 346, no. 8979, pp. 877-880, 1995.

[7] D. Lockey and D. N. Bateman, "Effect of oral activated charcoal on quinine elimination," British Journal of Clinical Pharmacology, vol. 27, no. 1, pp. 92-94, 1989.

[8] L. F. Prescott, A. R. Hamilton, and R. Heyworth, "Treatment of quinine overdosage with repeated oral charcoal," British Journal of Clinical Pharmacology, vol. 27, no. 1, pp. 95-97, 1989.

[9] A. Jaeger, P. Sauder, J. Kopferschmitt, and F. Flesch, "Clinical features and management of poisoning due to antimalarial drugs," Medical Toxicology and Adverse Drug Experience, vol. 2, no. 4, pp. 242-273, 1987.

[10] J.-L. Clemessy, P. Taboulet, J. R. Hoffman et al., "Treatment of acute chloroquine poisoning: a 5-year experience," Critical Care Medicine, vol. 24, no. 7, pp. 1189-1195, 1996.

[11] J. L. Clemessy, S. W. Borron, and F. J. Baud, "Hypokalaemia and acute chloroquine ingestion-reply," The Lancet, vol. 347, no. 8998, pp. 404-405, 1996.

[12] J. L. Crouzette, E. Vicaut, S. Palombo, C. Girre, and P. E. Fournier, "Experimental assessment of the protective activity of diazepam on the acute toxicity of chloroquine," Journal of Toxicology: Clinical Toxicology, vol. 20, no. 3, pp. 271-279, 1983.

[13] J. Demaziere, J. M. Saissy, M. Vitris et al., "Effects of diazepam on mortality from acute chloroquine poisoning," Annales Françaises d'Anesthèsie et de Rèanimation, vol. 11, no. 2, pp. 164167, 1992.

[14] J. Crouzette, E. Vicaut, S. Palombo, C. Girre, and P. E. Fournier, "Experimental assessment of the protective activity of diazepam on the acute toxicity of chloroquine," Journal of Toxicology: Clinical Toxicology, vol. 20, no. 3, pp. 271-279, 1983.

[15] F. Mongenot, Y. Tessier Gonthier, F. Derderian, M. Durand, and D. Blin, "Treatment of hydroxychloroquine poisoning with extracorporeal circulation," Annales Francaises d'Anesthesie et de Reanimation, vol. 26, no. 2, pp. 164-167, 2007.

[16] C.-Y. Chen, F.-L. Wang, and C.-C. Lin, "Chronic hydroxychloroquine use associated with QT prolongation and refractory ventricular arrhythmia," Clinical Toxicology, vol. 44, no. 2 , pp. 173-175, 2006.

[17] L. Rothschild, S. Bern, S. Oswald, and G. Weinberg, "Intravenous lipid emulsion in clinical toxicology," Scandinavian Journal of Trauma, Resuscitation and Emergency Medicine, vol. 18, no. 1, article 51, 2010.

[18] G. L. Weinberg, T. VadeBoncouer, G. A. Ramaraju, M. F. GarciaAmaro, and M. J. Cwik, "Pretreatment or resuscitation with a lipid infusion shifts the dose-response to bupivacaine-induced asystole in rats," Anesthesiology, vol. 88, no. 4, pp. 1071-1075, 1998.

[19] G. Weinberg, R. Ripper, D. L. Feinstein, and W. Hoffman, "Lipid emulsion infusion rescues dogs from bupivacaine-induced cardiac toxicity," Regional Anesthesia and Pain Medicine, vol. 28, no. 3, pp. 198-202, 2003.

[20] M. A. Rosenblatt, M. Abel, G. W. Fischer, C. J. Itzkovich, and J. B. Eisenkraft, "Successful use of a $20 \%$ lipid emulsion to resuscitate a patient after a presumed bupivacaine-related cardiac arrest," Anesthesiology, vol. 105, no. 1, pp. 217-218, 2006.

[21] R. J. Litz, M. Popp, S. N. Stehr, and T. Koch, "Successful resuscitation of a patient with ropivacaine-induced asystole after axillary plexus block using lipid infusion," Anaesthesia, vol. 61, no. 8, pp. 800-801, 2006.
[22] A. J. Sirianni, K. C. Osterhoudt, D. P. Calello et al., "Use of lipid emulsion in the resuscitation of a patient with prolonged cardiovascular collapse after overdose of bupropion and lamotrigine," Annals of Emergency Medicine, vol. 51, no. 4, pp. 412.el-415.el, 2008.

[23] H.-S. Lim, J.-S. Im, J.-Y. Cho et al., "Pharmacokinetics of hydroxychloroquine and its clinical implications in chemoprophylaxis against malaria caused by Plasmodium vivax," Antimicrobial Agents and Chemotherapy, vol. 53, no. 4, pp. 14681475, 2009.

[24] P. F. Kolecki and S. C. Curry, "Poisoning by sodium channel blocking agents," Critical Care Clinics, vol. 13, no. 4, pp. 829848, 1997.

[25] O. F. Wong, Y. C. Chan, S. K. Lam, H. T. Fung, and J. K. Y. Ho, "Clinical experience in the use of intravenous lipid emulsion in hydroychloroquine and chloroquine overdose with refractory shock," Hong Kong Journal of Emergency Medicine, vol. 18, no. 4, pp. 243-248, 2011. 


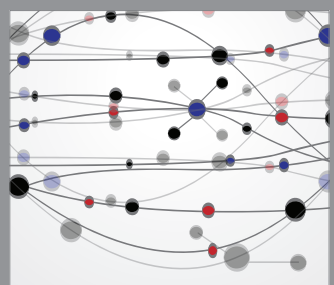

The Scientific World Journal
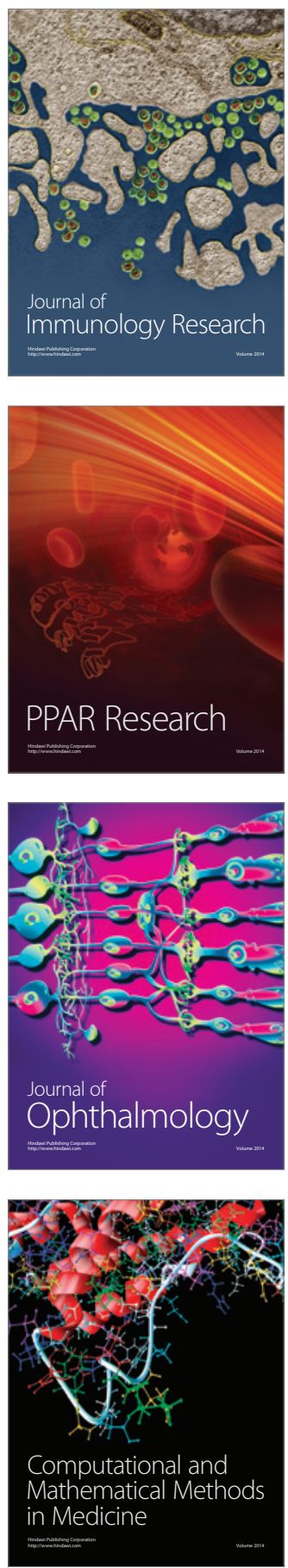

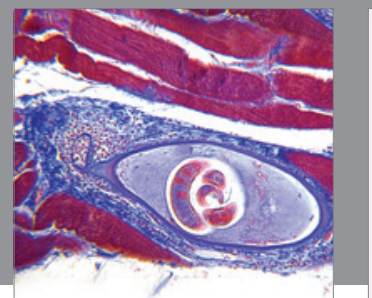

Gastroenterology

Research and Practice
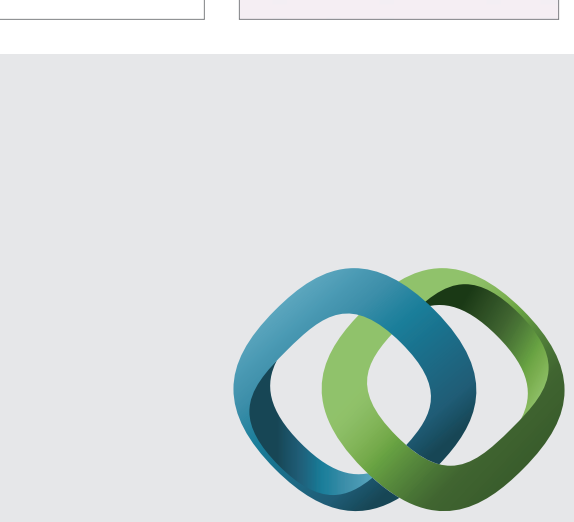

\section{Hindawi}

Submit your manuscripts at

http://www.hindawi.com
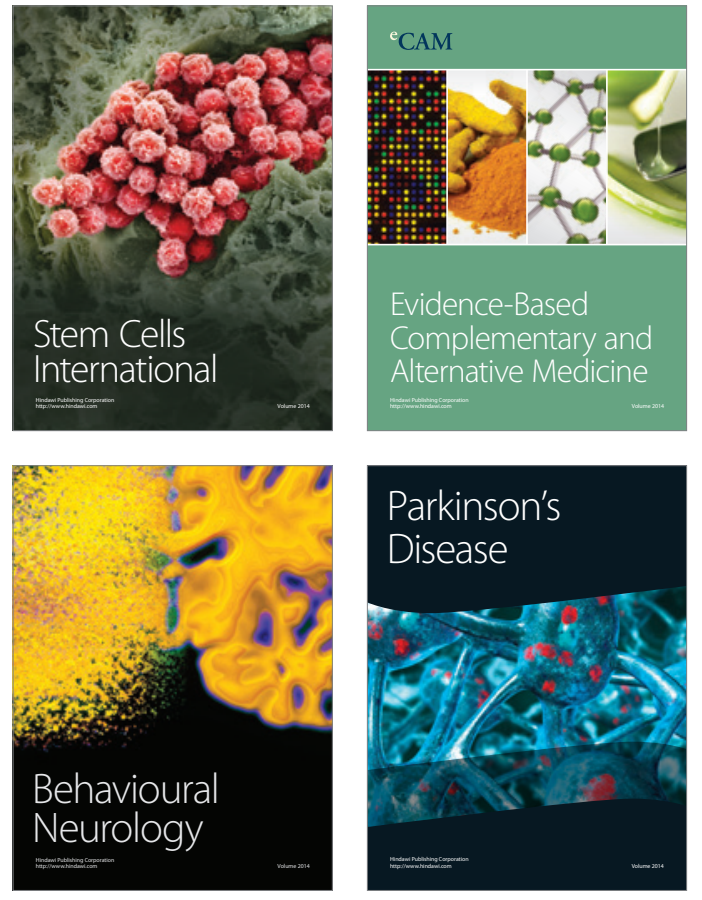
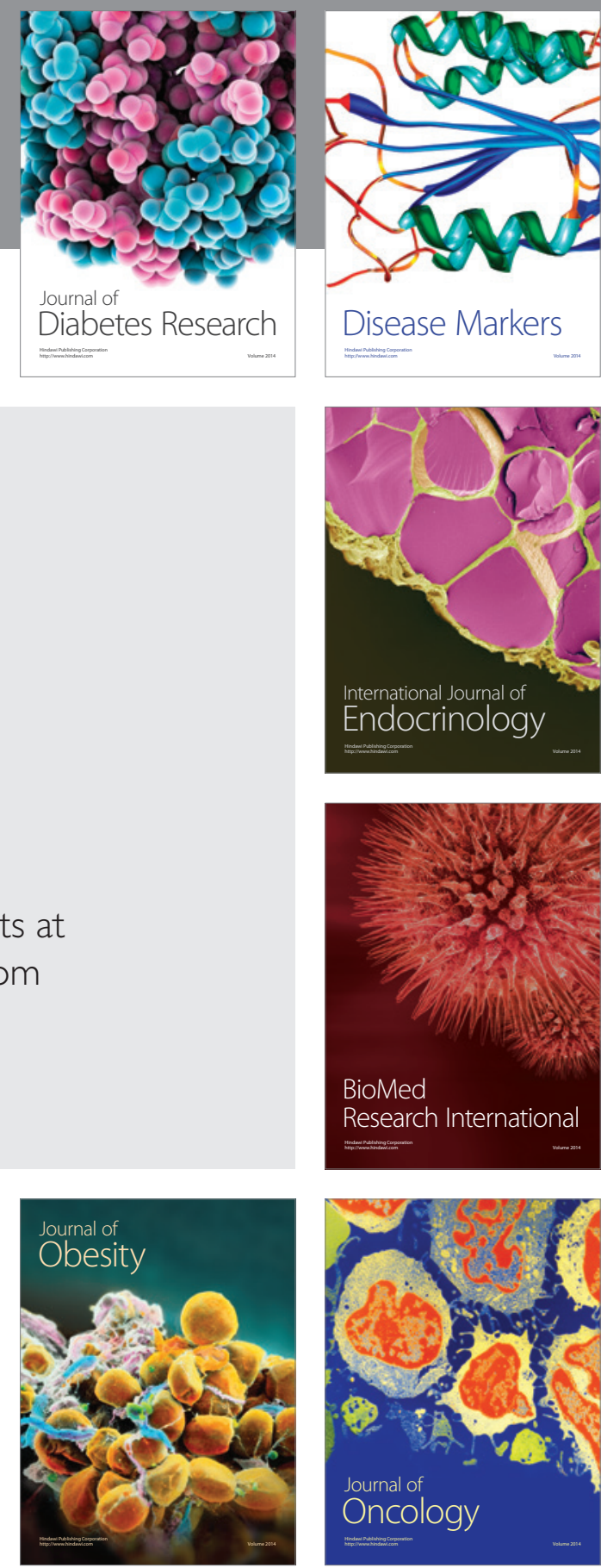

Disease Markers
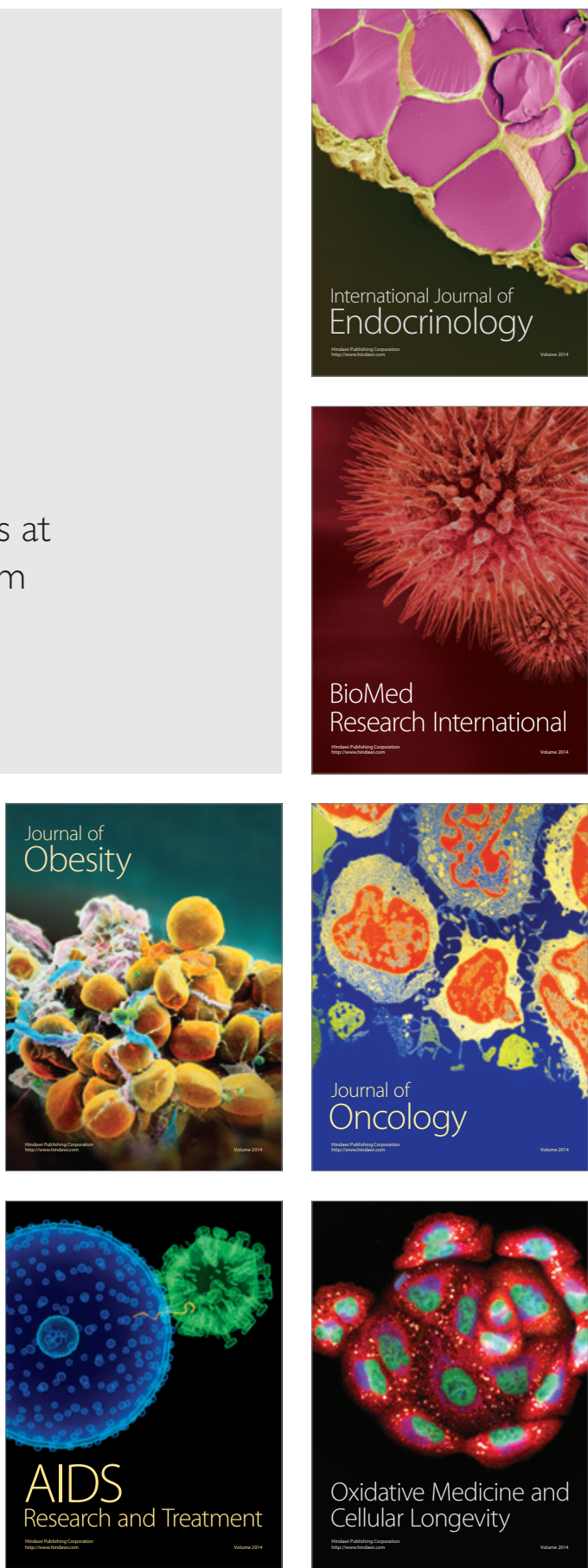